\title{
Implementation of IoT in Workplace Monitoring and Safety Systems
}

\author{
C.Annadurai ${ }^{\mathrm{a}, 1}$, I.Nelson ${ }^{\mathrm{b}}$, X.N.Ranald Nivethan ${ }^{\mathrm{b}}$, SurajVinod ${ }^{\mathrm{b}}$, M. Senthil Kumar ${ }^{\mathrm{b}}$ \\ ${ }^{\mathrm{a}, 1, \mathrm{~b}}$ Dept of ECE, SSN College of Engineering, Chennai, India
}

\begin{abstract}
The continuous and rapid development in facilities in the workplace eventually calls for safety of the workplace premises as well as improved monitoring system. For instance, an intruder alert will be sent even if a client enters the premises. To eradicate this issue, an alert notification has to be sent only when required i.e., during an intruder detection or mishap detection. The data is collected by Raspberry Pi using the sensors interfaced to it. By employing the usage of IoT, data received from the sensors are sent to an IoT platform from where the information is passed as a notification through an email. The detected face from the video recorded by PiCam is sent to a local server using socket programming and the Face recognition is performed in the local server using Haar cascade and LBPH algorithm in Open CV. In case of an intruder detection, an e-mail notification is sent to the user. Similarly, when an accident or disaster is detected such as a fire accident or air pollution, an alert notification is sent to the user through an e-mail.
\end{abstract}

Keywords: Internet of Things, Facial Recognition, Security System, Machine Learning.

\section{Introduction}

The need for safety and security is of utmost importance at present than ever before especially in a workplace or an industry. The word safety does not confine its concern only with the physical possessions of an organization but also an integrated protection. The rising need for safety has led to the emergence of various devices that serve the desired purpose. Initially products were designed to serve a single purpose and the majority of the devices were designed only to ensure the security of a workplace or an industry or even houses[2-4]. The vastly used product was the surveillance camera that helped people monitor their premises without the need for the presence of a human in place. This still remains as the largely preferred device for security but as far as the entirety of safety is concerned, an integrated safety system is the most suitable one. An integrated safety system helps in efficiently safeguarding the workplace in all areas possible. Recently, implementation of IoT in safety systems have been able to provide

\footnotetext{
${ }^{1}$ Anna DuraiC ,Dept of ECE, SSN college of Engineering, Chennai ,India E-mail: annaduraic@ssn.edu.in
} 
the user an easier access in monitoring their premises [1,5,6]. Although it has been helpful in many aspects there are still areas that can be further improved making the safety system even better. The system proposed here makes use of Picam and sensors such as smoke sensor and air quality sensor. This helps the user to know the state of their premises that includes features like intruder alert, smoke detection and air pollution detection.

\section{Proposed System}

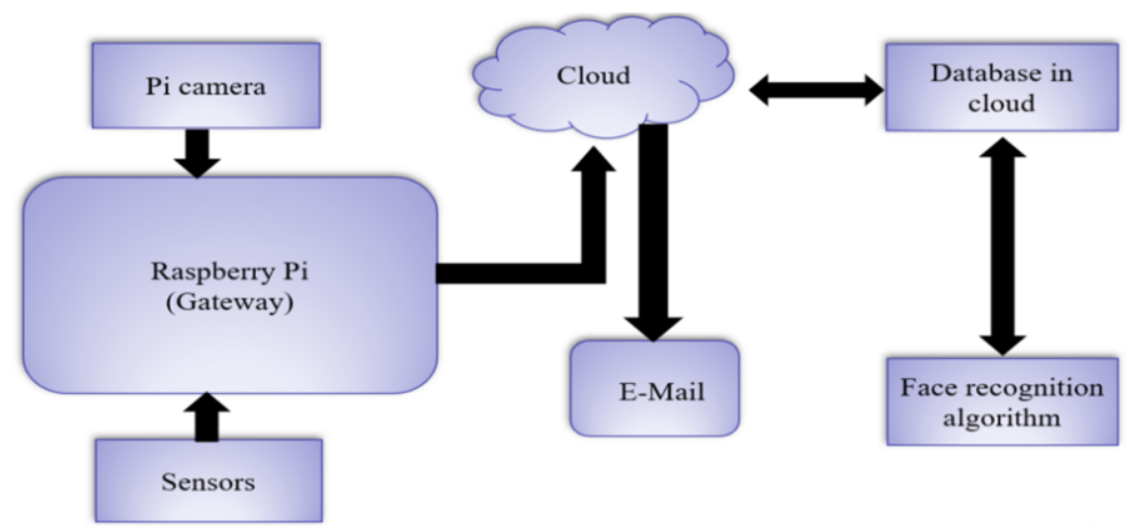

Figure 1. Block diagram of proposed system

\section{Face Detection Algorithm}

\subsection{Haar cascade}

Object Detection using Haar feature-based cascade classifiers is a constructive object detection method proposed by Paul Viola and Michael Jones in their paper, "Rapid Object Detection using a Boosted Cascade of Simple Features" in 2001.Based on positive and negative images, the cascade function will be trained. It is then used to identify objects in other images. At first, the algorithm should be provided with a lot of positive images (images of faces) and negative images (images without faces) to train the classifier. Based on the training, features are extracted from it. The sum of pixels of the white rectangle from the pixel sums is subtracted from black rectangle to obtain a feature. A variety of sizes and locations of each kernel are used to evaluate and capture lots of features. Using the integral image, the sum of pixels under black and white rectangle can be calculated for obtaining the features. The catch here is that, among all these features calculated, most of them are irrelevant. So, among the available features, the best features are selected using Adaboost algorithm.

All available features are applied on training images. One feature that strongly separates the positive images and negative images, alone is selected. Among the selected features, there will be errors and misclassifications as well. So, a feature which has the least error rate which classifies whether there is a face or no face is present is selected for use. By taking weighted sum of the classifiers which were selected from the previous iterations, we form a strong classifier. The classifier selected in previous stage 
alone cannot detect the images but by taking weighted average they can. Most of the pictures given does not contain a face. By using a check method, classify if a window consists of facial region or not and discard it in first time processing of the window and do not process it further. The rest of the region consists of facial region which can be focussed on instead of the entire image using classifiers in cascade. All the features selected are not applied in a single window, instead they are grouped into various stages. If a window does not successfully pass through one stage, it is discarded and it is not processed in further layers. If the same window passes in one stage then the next stage of features is applied. If the same window passes through all stages, then it is considered as a face region.

\subsection{Local binary pattern histogram(Lbph)}

Local binary pattern designates a particular pixel of an image by using the binary number that we got from the threshold levels of the neighbouring pixels of the current pixel. This strategy is very simple as well as efficient. The local binary pattern uses four parameters for thresholding the neighbourhood pixels, they are: radius, neighbours, grid $\mathrm{x}$ and grid $\mathrm{y}$. The radius is used to represent the area around the chosen pixel and subsequently used to construct the binary pattern. The neighbours is the count of the sample points required to build the binary pattern corresponding to the radius chosen. When the count of the sample points obtained is more, then the computational resources needed is also higher. The grid $\mathrm{x}$ parameter is used to find the number of cells present in the horizontal direction. The feature vector's dimension depends on this parameter. If the dimension is more, the grid is also more fine. The grid y parameter is used to find the number of cells present in the vertical direction. The feature vector's dimension increases as the grid becomes more fine. The algorithm is trained using the data of the people who will be recognized using their face images. Each person's image is given an ID(identification) which the algorithm uses to give output corresponding to an input image. All images of a particular person are given the same ID. By using the sliding window algorithm that takes neighbours and radius parameters into account, the algorithm creates an intermediate image that highlights the facial characters and represents the given image in a better way. The image given is converted in grayscale and a portion of this image as a window of $3 \times 3$ pixels is obtained. This is represented in a matrix that has intensity of each pixels. By using the value that is middle element in the matrix as threshold, the neighbours are given a binary value depending on whether they are greater than or less than the threshold. This matrix is assigned a new binary value by concatenating each binary value from each position. The value obtained is then converted into a decimal value and set to the central value of the matrix which is a new pixel value from the original image. Thus, the obtained image represents the characteristics of the original image in a better way.

Grid $\mathrm{X}$ and grid $\mathrm{Y}$ parameters are used to divide the image into multiple grids each represented as a histogram containing 256 positions representing the occurrences of each pixel intensity. A final histogram representing the characteristics of the original image is obtained by concatenating the individual histograms. A new image is captured and the same steps above are performed for Face recognition. Now the obtained histograms are compared with existing histograms using Euclidean distance. Therefore the output of the algorithm is the ID of the most matching histogram. 


\section{Result}
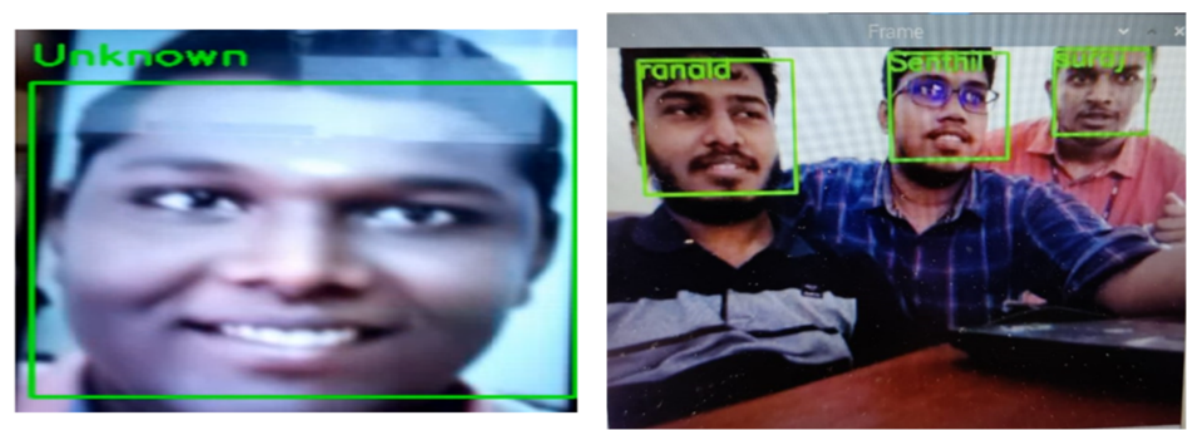

Figure 2. Output with known and unknown perrsons

\section{Conclusion}

The proposed system is designed in such a way that it integrates multiple features such as surveillance, disaster and accident management into a single system. The compactness and simplicity of the system makes it easy to use for the user. As far as disaster and accident management is concerned, the system sends an alert notification in case of any mishap which helps the user be able to be aware of the status of the premises even when not present. The surveillance system employed is made efficient by using smart recording feature and by avoiding unnecessary intruder alerts. The unnecessary intruder alerts is eliminated by sending an e-mail to the user and he decides if the person is an intruder or not. Thus, the proposed system is more useful and easy to operate. This also increases the comfort of the user.

\section{References}

[1] Sonali P. Gulve, Suchitra A. Khoje, PrajaktaPardeshi, Implementation of IoT-based Smart Video Surveillance System, Conference: Computational Intelligence in Data Mining, At: Singapore Vol: 556, 2017

[2] Sun, Yi, Xiaogang Wang, and Xiaoou Tang, Hybrid Deep Learning for Face Verification IEEE Transactions on Pattern Analysis and Machine Intelligence Vol 38, No 102013

[3] R. Chellappa, C.L. Wilson and S. Sirohey, Human and machine recognition of faces: A survey, Proc. IEEE , Vol 83, pp. 705-740, 1995

[4] Paul Viola, Michael Jones, Rapid Object Detection using a Boosted Cascade of Simple Features, Accepted Conference on Computer Vision and Pattern Recognition 2001.

[5] Ishita Gupta, Mrs.VarshaPatil, ChaitaliKadam, ShreyaDumbre, Face detection and Recognition using Raspberry pi”, IEEE International WIE Conference 185 on Electrical and Computer Engineering (WIECON-ECE), pages 83-86. IEEE, dec 2016

[6] PravinKumar.S, Bhuvaneswari Balachander, Fast and Cost Efficient Face Detection System with CNN Using Raspberry PI , International Journal of Engineering and Advanced Technology (IJEAT) ISSN: 2249 - 8958, Volume-8 Issue-6, August, 2019.

[7] V.D.Ambeth Kumar et.al, , "FACIAL RECOGNITION SYSTEM FOR SUSPECT IDENTIFICATION USING A SURVEILLANCE CAMERA”, Pattern Recognition and Image Analysis (Springer), Volume 28, Issue 3, pp 410-420, 2018. (DOI: 10.1134/S1054661818030136)

[8] V.D.Ambeth Kumar (2017), "Efficient Routing for Low Rate Wireless Network a Novel Approach", International Journal of Image Mining, Vol. 2, Nos. 3/4, 2017, 2017. 